\title{
EDITORIAL
}

\section{Cost of tuberculosis in the era of multidrug resistance: will it become unaffordable?}

\author{
Robert Loddenkemper*, Giovanni Sotgiü and Carole D. Mitnick
}

I n 1905, Robert Koch ended his Nobel Lecture on "The current state of the struggle against tuberculosis" with the optimistic sentence: "If the work goes on in this powerful way, then the victory must be won" [1]. At the end of the 1970s and the beginning of the 1980s, many believed that tuberculosis (TB) was nearly vanquished [2]. Now, more than 100 years after Koch's Nobel Lecture, TB has emerged as an even greater public health problem, mainly for two reasons: coinfection with HIV and the development of complex mycobacterial drug resistance patterns [3].

The World Health Organization (WHO) estimates that of the 8.8 million new cases in 2010, $3 \%$ were caused by multidrugresistant (MDR) strains of Mycobacterium tuberculosis [4], defined as resistance to at least the two most powerful anti-TB drugs, isoniazid and rifampicin. Furthermore, $\sim 30,000$ cases were thought to be due to extensively drug-resistant (XDR) strains, defined as MDR plus resistance to any fluoroquinolone and at least one second-line injectable anti-TB drug (amikacin, capreomycin or kanamycin). The estimated prevalence of MDR-TB in new and previously treated cases in 2010 was 650,000 worldwide [4].

MDR- and XDR-TB are man-made phenomena that emerge as a result of inadequate treatment of $\mathrm{TB}$ and/or poor airborne infection control in healthcare facilities and congregate settings [5]. To resolve the epidemic of MDR-TB, several interventions are needed urgently: rapid case detection, proper infection control, timely access to quality-assured first- and second-line drugs within appropriate regimens, capacity-building to deliver treatment effectively, standardised recording and reporting of treatment outcomes [6] within effective national TB control programmes, and the commitment of national governments [7].

Nine of the countries with the greatest MDR-TB burden worldwide are located in the WHO European Region, which had, in 2009, an estimated 81,000 MDR-TB patients [5]. The highest proportions of MDR-TB, up to $26 \%$ and $65 \%$ among new and previously treated cases, respectively, are seen in the countries of the former Soviet Union (FSU), with an estimated 66,000 reported cases in total. However, less than one-third are

\footnotetext{
*German Central Committee Against Tuberculosis, Berlin, Germany. ${ }^{\#}$ Epidemiology and Medical Statistics Unit, Dept of Biomedical Sciences, University of Sassari, Sassari, Italy. "Dept of Global Health and Social Medicine, Harvard Medical School, Boston, MA, USA.

CORRESPONDENCE: R. Loddenkemper, German Central Committee Against Tuberculosis, Stralauer Platz 34, 10243 Berlin, Germany. E-mail: rloddenkemper@dzk-tuberkulose.de
}

diagnosed throughout the European Region because of limited access to the new WHO-approved rapid diagnostic methods [5].

Treatment of MDR-TB (and even more of XDR-TB) is complicated, expensive and often unsuccessful, with low cure and high mortality rates [6]. Only $2-3 \%$ of an estimated global prevalence of 1-1.5 million MDR-TB cases was known to be treated according to $\mathrm{WHO}$ recommendations [8].

To date, there is no clear scientific evidence focused on the economic burden of MDR/XDR-TB management in the WHO European Region. Health economic analyses could be used to estimate the value and the economic impact of different healthcare interventions in order to adequately allocate public money and resources. Yet, over the last few decades, private companies and policymakers working in the public sector have been adopting health economic evaluations (i.e. Health Technology Assessment) to increase the efficacy and the efficiency of their choices and decisions as one way to respond to perceived scarcity in global resources for equitable healthcare.

Applying this methodology to the aforementioned scientific gap, this issue of the European Respiratory Journal (ERJ) includes two relevant economic studies focused on drug-resistant TB in Europe.

The study by FloYD et al. [9], carried out in Estonia and Russia (Tomsk Oblast), both middle-income countries, compared the cost of MDR-TB treatment before and after the introduction of WHO guidelines. Furthermore, the authors evaluated the costeffectiveness of this treatment, using cure rates, deaths averted and disability-adjusted life years (DALYs) averted as outcomes. It follows the outline of two WHO economic studies performed in non-European countries, those previously reported from Peru [10] and the Philippines [11].

Their findings highlight that treatment success increased from $15 \%$ to $76 \%$ in Tomsk Oblast and from $52 \%$ to $61 \%$ in Estonia; the death frequency fell from $64 \%$ to $4 \%$ in Tomsk Oblast and from $24 \%$ to $13 \%$ in Estonia. After the introduction of the WHO guidelines in 2001 and 2002, the majority of MDR-TB patients enrolled in Estonia had a high degree of drug resistance $(79 \%$ were resistant to five or more anti-TB drugs or XDR versus $28 \%$ in Tomsk Oblast), whereas in Tomsk Oblast, stricter enrolment criteria were applied with priority for treatment given to more seriously ill patients, due in part to shortages of drugs during the study period. After the introduction of the guidelines, the WHO Green Light Committee supported the supply of secondline drugs at the lowest possible price to both countries. 
The average cost per patient treated for MDR-TB almost doubled in Estonia, from US\$4,729 to US\$8,974, and in Tomsk Oblast it increased four- to five-fold, from US\$2,282 to US $\$ 10,088$, mainly due to higher costs for in-patient care (related to higher income levels) and for drugs, which together accounted for $69-90 \%$ of total costs. The net increase in total costs was about US $\$ 0.5$ million in Estonia and US\$1.0 million in Tomsk Oblast. These additional costs of treatment according to WHO guidelines resulted in a large number of averted deaths and DALYs, the cost per DALY averted being US\$400-US\$600, and thus provide clear evidence for the cost-effectiveness of the new treatment programme according to the WHO guidelines. In 2010, due to the increase in the gross national income per capita, the estimated costs per patient would reach US\$14,370 in Estonia and US\$9,910 in Russia.

Although the data from this study have been collected from cohorts enrolled in 2001-2002, these are contemporaneous with other publications. In Peru [10] and the Philippines [11], the costs for treating one MDR-TB patient were calculated at US\$2,381 and US\$3,355, respectively, mainly because hospitalisations were avoided to a large degree, and, when used, the cost of hospitalisation was lower than in the European countries. By comparison, RAJBHANDARY et al. [12] calculated substantially higher costs for the USA. On the basis of an analysis of 13 MDR-TB patients in three different illness severity categories, the average direct (in-patient and outpatient) costs per patient amounted to US $\$ 44,881$ (range US $\$ 12,495-U S \$ 115,393$ ) plus indirect costs due to productivity loss at an average of US\$32,964 (range US\$9,208-US\$66,099) for those who survived and US\$686,381 (range US\$496,995US\$1,256,395) for those who died.

These data for direct costs are quite consistent with the data from Germany reported by DIEL et al. [13] in this issue of the $E R J$. In their analysis, the authors estimate direct (combined inpatient/outpatient) costs of $€ 52,259$ (at the time of writing of this editorial, $€ 1 \sim$ US\$1.3) per MDR-TB patient, which are much higher than the costs in Estonia and Russia. This is probably due to substantially higher wages and drug prices in Germany. However, these costs may even be underestimated. Although the number of MDR-TB cases was relatively small (in 2009,63 cases, i.e. $2.1 \%$ of all cases with susceptibility testing or $1.4 \%$ of all 4,444 cases), they significantly contributed $(8.5 \%)$ to the total treatment costs of almost US\$50 million for TB treatment in Germany. Thus, TB must still be classified in Germany, with its low incidence rate of 5.4 cases per 100,000 population, as a disease of economic significance. As a country located not far from the countries of the FSU, the number of MDR-TB (and XDR-TB) cases may possibly rise in the future. $\mathrm{XDR}-\mathrm{TB}$ patients were not included in the present cost analysis (in 2004-2006, the costs of treating XDR-TB patients in Germany amounted up to more than $€ 170,000$ per patient [14]) as well as possible surgical interventions, expensive second-line anti-TB drugs such as linezolid and HIV coinfection, since these factors play only a marginal role in the German scenario at present.

Another interesting finding described in the study by DiEL et al. [13] is related to the lower direct costs of treating drugsensitive TB patients, which amounted to $€ 7,364$ in adults and
$€ 7,300$ in children in 2009 , compared with the higher costs computed in 2001 ( $€ 14,301$ and $€ 16,634$ in adults and children, respectively). This decrease is explained mainly by the reduction in hospitalisations (from $80.0 \%$ to $71.2 \%$ ) plus the considerably reduced length of hospitalisation (from a mean of 50 days to 30 days). The comparison of costs between treatments for drug-sensitive TB and MDR-TB highlights the disproportionate contribution of drugs and hospitalisation to the overall cost for MDR-TB treatment $[9,13]$, as noted above. The drug costs for MDR-TB among the studies mentioned represent from just over 30\% (Peru and the Philippines) and as much as $50 \%$ (Germany) of the total treatment costs. In all cases, the costs of drugs for treatment of MDR-TB are considerably higher than those for drug-susceptible TB; in the report by DiEL et al. [13], almost 50 times high. This is, in part, due to the extended duration of MDR-TB treatment (three to four times as long as treatment for drug-susceptible TB). But cure rates are considerably lower, estimated at $62 \%$ in one recent meta-analysis of MDR-TB treatment compared with $85-90 \%$ for treatment of drug-sensitive TB [15]. These observations underscore the urgent need for new drugs for the treatment of MDR-TB and improved production and distribution of existing drugs, many of which remain very expensive, despite being off patent [6].

Similarly, the cost of hospitalisation, in places where it was routine, comprised between 30\% (Estonia under WHO guidelines) and 51\% (Tomsk Oblast under WHO guidelines) of total treatment costs. Moreover, the overall cost of MDR-TB treatment in those places was five to 30 times more than treatment in places where routine care was ambulatory (Peru and the Philippines). This increase in cost was not matched by significant improvement in effectiveness; a recent report by FITZPATRICK and FLOYD [16] revealed that the cost per DALY averted in Peru and Philippines was one-quarter to one-fifth that of the cost in Estonia and Tomsk Oblast.

FLOYD et al. [9] extrapolate on the basis of results from Tomsk Oblast that the annual cost of MDR-TB treatment for the whole of Russia would amount to US\$375 million. This would already require a substantial increase in funding for TB control in Russia. Since India and China have much higher numbers of MDR-TB cases, with about half of the world's estimated cases, the cost of MDR-TB control alone in these two countries would require, even with the low costs achieved in the Philippines and Peru, enormous investments. Thus, if the tide cannot be reversed in the near future, with new drugs and diagnostics, as well as broader introduction of more cost-effective models of care, global MDR-TB control may become unaffordable.

The countrywide evaluation of the costs and benefits of policies, guidelines and diagnostic/treatment practices represents a new tool for the management of TB, drug-resistant TB and TB/HIV co-infection in the near future. Moreover, the current economic crisis demands innovation in the development of tools as well as in decision-making processes for the introduction (or elimination) of useful (or harmful) healthcare approaches, tools and activities. Cost analysis, cost-effectiveness and cost-benefit studies provide useful insight into the efficiency of novel healthcare interventions and highlight opportunities for improvements in tools and care models that could improve effectiveness. 


\section{STATEMENT OF INTEREST}

None declared.

\section{REFERENCES}

1 Koch R. The Current State of the Struggle against Tuberculosis. www.nobelprize.org/nobel_prizes/medicine/laureates/1905/kochlecture.html

2 Dheda K, Migliori GB. The global rise of extensively drug-resistant tuberculosis: is the time to bring back sanatoria now overdue? Lancet 2012; 379: 773-775.

3 Raviglione MC, Uplekar MW. WHO's new Stop TB Strategy. Lancet 2006; 367: 952-955.

4 World Health Organization. Global Tuberculosis Control Report 2011. Geneva, Switzerland, 2011.

5 World Health Organization. Consolidated action plan to prevent and combat multidrug- and extensively drug-resistant tuberculosis in the WHO European Region 2011-2015. www.euro.who.int/_data/ assets /pdf_file/0007/147832/wd15E_TB_ActionPlan_111388.pdf Date last updated: July 21, 2011.

6 Keshavjee S, Farmer P. Picking up the pace - scale up of MDR tuberculosis treatment programs. N Engl J Med 2010; 363 1781-1784.

7 Trébucq A, Enarson DA, Chiang CY, et al. Xpert MTB/RIF for national tuberculosis programmes in low-income countries: when, where and how? Int J Tuberc Lung Dis 2011; 15: 1567-1572.
8 World Health Organization. 2011/2012 Tuberculosis Global Facts. Geneva, Switzerland, 2011.

9 Floyd K, Hutubessy R, Kliiman K, et al. Cost and cost-effectiveness of multidrug-resistant tuberculosis treatment in Estonia and Russia. Eur Respir J 2012; 40: 133-142.

10 Suárez PG, Floyd K, Portocarrero J, et al. Feasibility and costeffectiveness of standardised second-line drug treatment for chronic tuberculosis patients: a national cohort study in Peru. Lancet 2002; 359: 1980-1989.

11 Tupasi TE, Gupta R, Quelapio MID, et al. Feasibility and costeffectiveness of treating multidrug resistant tuberculosis: a cohort study in the Philippines. PLoS Medicine 2006; 3: e352.

12 Rajbhandary SS, Marks SM, Bock NN. Costs of patients hospitalized for multidrug-resistant tuberculosis. Int J Tuberc Lung Dis 2004; 8: 1012-1016.

13 Diel R, Rutz S, Castell S, et al. Tuberculosis: cost of illness in Germany. Eur Respir J 2012; 40: 143-151.

14 Blaas SH, Mütterlein R, Weig J, et al. Extensively drug-resistant tuberculosis in a high-income country: a report of four unrelated cases. BMC Infect Dis 2008; 8: 60.

15 Orenstein EW, Basu S, Shah NS, et al. Treatment outcomes among patients with multidrug-resistant tuberculosis: systematic review and meta-analysis. Lancet Infect Dis 2009; 9: 153-161.

16 Fitzpatrick C, Floyd K. A systematic review of the cost and cost effectiveness of multidrug-resistant tuberculosis. Pharmacoeconomics 2012; 30: 63-80. 